\title{
MODELO PARA MELHORIA DE RESULTADOS NO SETOR DE MANUTENÇÃO DE UMA INDÚSTRIA TÊXTIL ATRAVÉS DO MÉTODO ADITIVO COM VETO
}

\author{
Hugo Augusto (UNIFAVIP | WYDEN) hugo_augusto07@hotmail.com \\ Matheus Valeriano (UNIFAVIP | WYDEN) valeriano1214@hotmail.com \\ Jean Turet (UNIFAVIP | WYDEN) jeanturet@gmail.com
}

\begin{abstract}
Resumo
Problemáticas envolvendo o setor de manutenção nas organizações vem se tornando ao longo dos tempos cada vez mais complexas, fazendo com que os gestores necessitem investir e planejar de forma adequada, para que estes problemas não afetem a produtividade e a qualidade dos produtos nas organizações. Dessa forma, uma empresa que tem um bom planejamento e gestão de manutenção sai na frente dos seus concorrentes, pois terá um controle eficiente do estoque de reposição de peças e também escolherá o tipo de manutenção que mais se encaixa a sua realidade, fazendo com que tenha o foco de reduzir a quantidade de tempo parado do seu maquinário. O presente trabalho se propõe a utilizar a metodologia de Apoio à Decisão Multicritério, com aplicação do método aditivo com veto, para a priorização, proposta por De Almeida (2013), auxiliando no processo decisório de melhoria de resultados do setor de manutenção em uma indústria do setor têxtil em Pernambuco. Como resultado, tem-se um modelo em que empresas podem utilizar para priorizar as suas ações nesse setor.
\end{abstract}

Palavras-Chaves: Apoio a Decisão Multicritério, Aditivo com Veto, Indústria, Manutenção.

\section{Introdução}

Na busca pela elevação dos níveis produtivos e competitivos no mercado atual, as organizações necessitam de um sistema de planejamento e controle de manutenção extremamente eficaz. Problemas como tempo parado por quebras ou falta de itens em estoque para reposição de máquinas, fazem com que a produtividade e lucratividade de empresas sejam diretamente afetados. Por isso, para uma organização ser bem sucedida é de grande importância uma manutenção voltada na confiabilidade máxima do seu maquinário, pois quanto maior o tempo entre falhas do seu maquinário mais eficiente será o sistema produtivo da organização.

A manutenção trata-se da combinação de todas as ações necessárias para conservar um objeto ou para o restabelecê-lo, de modo que este possa executar a sua função da melhor forma possível. Deste modo, os objetivos principais, dentro da manutenção, são: assegurar o funcionamento do sistema e garantir o bom desempenho dos equipamentos ao longo da sua vida útil. De acordo com a ABNT no padrão NBR-5462 [1994], manutenção é definida como o conjunto de atividades administrativas, de supervisão e técnicas que tem por objetivo manter a integridade ou reposicionar um equipamento de forma que ele continue desempenhando a função que lhe é desejada.

Escolher o tipo de manutenção a se utilizar em uma respectiva organização é de fundamental relevância, pois caso o tipo escolhido se encaixe bem na realidade da empresa, fará com que a 
confiabilidade e utilidade do maquinário utilizado na organização seja alavancada. De acordo com uma pesquisa realizada pela ABRAMAN (Associação Brasileira de Manutenção e Gestão de Ativos), em 2013, os tipos de manutenção adotados nas empresas brasileiras estão distribuídos conforme Tabela 1.

Tabela 1 - Pesquisa sobre tipos de manutenção na indústria brasileira

\begin{tabular}{|c|c|c|c|c|}
\hline Ano & $\begin{array}{c}\text { Manutenção } \\
\text { Corretiva }\end{array}$ & $\begin{array}{c}\text { Manutenção } \\
\text { Preventiva }\end{array}$ & $\begin{array}{c}\text { Manutenção } \\
\text { Preditiva }\end{array}$ & Outros \\
\hline 2013 & 30,86 & 36,55 & 18,82 & 13,77 \\
\hline
\end{tabular}

Fonte - http://www.abraman.org.br/Arquivos/403/403.pdf

No entanto, cada empresa apresenta uma realidade diferente e cabe aos encarregados pelo setor de manutenção ou a empresa terceirizada responsável, escolher a melhor estratégia e tipo de manutenção a se utilizar na sua respectiva organização.

Dentro desta realidade, o presente artigo tem como objetivo propor a elaboração de um modelo multicritério de apoio à decisão, utilizando-se do método aditivo com veto, objetivando a melhoria dos resultados do setor de manutenção de uma empresa do setor têxtil localizada no agreste pernambucano.

\section{Desafios}

$\mathrm{Na}$ linha de obter um maior tempo entre falhas de máquinas, as indústrias direcionam suas atenções para melhorar as condições de trabalho dos seus equipamentos. As possibilidades variam de acordo com a situação de cada empresa, desde sua realidade interna até a área de atuação; cuja aplicação deve ser analisada de forma coerente para obter um melhor aproveitamento dos recursos disponibilizados.

O senso comum julga a tecnologia como o meio mais rápido para conquistar resultados a curto prazo, porém existem particularidades na área da manutenção que fornecem oportunidades de melhoria de forma mais simples e eficaz. Nesse sentido, os desafios apontados nesse setor estão apresentados na Tabela 2 .

Tabela 2 - Desafios encontrados na empresa em estudo

\begin{tabular}{c|c|c}
\hline ID & Desafios & Descrição \\
\hline \hline D1 & Manutenção Preventiva & $\begin{array}{c}\text { Não existe um programa de Manutenção Preventiva dentro da } \\
\text { empresa }\end{array}$ \\
\hline D2 & Catálogo das Máquinas & A listagem com todos os itens das máquinas é inexistente \\
\hline
\end{tabular}




\begin{tabular}{c|c|c}
\hline D3 & Peças Disponíveis & As peças mais utilizadas não possuem um estoque mínimo \\
\hline D4 & Comunicação Interna & Os colaboradores da Manutenção não dialogam entre si \\
\hline D5 & Compra de Peças & $\begin{array}{c}\text { Itens necessários para as máquinas não possuem cadastro no } \\
\text { sistema e compras não possuem prazo de conclusão }\end{array}$ \\
\hline
\end{tabular}

Fonte - Os Autores (2020)

\section{Estudos Relacionados}

Para uma organização se manter ativa é de grande importância que o seu maquinário e seus equipamentos mantenham um bom desempenho dentro da sua vida útil, a manutenção é um setor crucial para que as empresas atendam os padrões de qualidade exigidas pelos seus respectivos consumidores. Slack (2000) definiu manutenção como o termo usado para abordar a forma pela qual as organizações tentam evitar as falhas ao cuidar de suas instalações físicas. Hoje a missão da manutenção é garantir a disponibilidade da função dos equipamentos e instalações de modo a atender um processo de produção ou serviço, com confiabilidade, segurança, preservação do meio ambiente e custos adequados. (KARDEC; NASCIF, 1998).

Por isso, falar de manutenção hoje não é somente falar em reparo de máquinas, mas sim na qualidade e imagem que a empresa passa para o mercado, como também do modo que esses equipamentos estão condizentes com as políticas ambientais presentes.

Outro ponto importante na manutenção é o gerenciamento de estoque de peças para o reparo de máquinas, caso uma organização tenha um estoque mínimo para peças de reparo é um controle eficiente do que entra e sai neste mesmo estoque, esta empresa terá a sua gestão da manutenção muito mais facilitada. A gestão de peças sobressalentes influencia positivamente no gerenciamento de manutenção, uma vez que leva a uma maior confiabilidade e disponibilidade de equipamentos e, portanto, tem um impacto direto na rentabilidade do negócio (ALMEIDA et al., 2015).

Por isso, manutenção hoje em dia não é somente o reparo de maquinas ou equipamentos da forma mais rápida possível, mas sim todo um estudo sobre a realidade da empresa e toda uma gestão e planejamento de manutenção, afim de controlar o estoque de reparo de peças da melhor forma possível e também escolher e gerenciar o melhor tipo de manutenção, para que este seja executado da maneira mais eficiente possível.

\section{Metodologia}

Este artigo tem como foco compreender os desafios encontrados no setor de manutenção de uma empresa têxtil no agreste pernambucano, para então propor alternativas que melhorem a 
situação encontrada, bem como sua ordenação de execução pautada no método aditivo com veto, fazendo com que os resultados possam ser atingidos com mais eficácia e a confiabilidade neste setor aumente e contribua com os anseios do decisor.

Para (Gil, 2008) A Pesquisa Bibliográfica é desenvolvida com base em material já elaborado, constituído principalmente de livros e artigos científicos. A fundamentação teórica do presente trabalho foi realizado através de levantamentos bibliográficos e também de trabalhos já publicados na área de multicritério, modelo aditivo com veto e manutenção.

O seguinte trabalho, também pode ser classificado como uma pesquisa aplicada, pois o modelo proposto pode ser replicado nas organizações que sofrem com problemas semelhantes ao apresentado no trabalho. A Pesquisa aplicada tem como objetivo os problemas presentes nas atividades das instituições, organizações, grupos ou atores sociais. Ela está empenhada na elaboração de diagnósticos, identificação de problemas e busca de soluções. (Thiollent, 2009). As metodologias utilizadas na resolução do problema abordado no trabalho são da área de pesquisa operacional que para Shamblin e Stevens Jr (1979, p. 13), Pesquisa Operacional ou (PO) é "um método científico de tomada de decisão". Com isso a seguinte problemática também se encaixa na perspectiva da abordagem quantitativa que segundo (SANTOS, 2000) é aquela pesquisa onde é importante a coleta e a análise quantificada dos dados, e, de cuja quantificação, resultados automaticamente apareçam. Qualitativa é aquela pesquisa cujos dados só fazem sentido através de um tratamento lógico secundário, feito pelo pesquisador.

Assim, um modelo multicritério de apoio à decisão foi realizado, sempre em consonância com o método escolhido (aditivo com veto) para possibilitar o exercício da aplicação numérica e comprovar a aplicação do modelo.

\section{Análise de Decisão Multicritério e Agregação Aditiva com Veto}

\subsection{Decisão Multicritério}

No contexto atual os gestores das organizações necessitam tomar decisões que vão influenciar muito na qualidade ou não dos seus respectivos produtos ou serviços, esse processo decisório pode estar relacionado a métodos formais e bem estruturados, a modo de trazer uma segurança maior para os gestores na resolução dos problemas. Por isso, ao tratar de cenários utilizando métodos de tomada de decisão com múltiplos critérios, segundo Barbosa, Santos e Gomes (2019), busca-se, geralmente, um apoio na complexidade de selecionar fatores qualitativos e quantitativos marcados por alvos múltiplos e conflitantes, habitualmente de difícil medição. Ainda de acordo com Gomes et al. (2011), a abordagem multicritério 
considera todas as possibilidades de resultado, permite estruturar problemas complexos para simplificar a resolução, estimula a participação ativa dos decisores e possibilita o modo de se trabalhar com as subjetividades e incertezas do processo decisório.

Com isso, os métodos multicritério de apoio a decisão, apresentam uma forma mais transparente de avaliação, a introdução dos critérios quanti e qualitativos e em alguns casos específicos, havendo trade-off entre eles (Moreira; Santos; Gomes, 2019).

No entanto, para a escolha do melhor modelo multicritério é de extrema relevância o gestor conhecer bem as necessidades e gargalos da sua organização, com o propósito de encaixar o problema e suas respectivas soluções da melhor forma possível. A escolha do método multicritério mais apropriado depende de diversos fatores considerados durante a fase de estruturação do problema. Por isso, no processo de escolha do método multicritério de apoio a decisão deve-se considerar as características do problema, o contexto, a estrutura de preferência do decisor, e o tipo de problemática (DE ALMEIDA; COSTA, 2003).

Com isso pode se classificar os métodos desenvolvidos de apoio a tomada de decisão em: Critério único de síntese, sobreclassificação e interativos (ROY, 1996; PARDALOS et al. 1995; KEENY \& RAIFFA, 1976). Por isso, é de grande importância e impacto para o processo decisório nas empresas o conhecimento não só dos problemas e suas respectivas soluções, mas sim, o método de apoio a tomada de decisão que mais se encaixa com a realidade do problema.

\subsection{Método de Agregação Aditiva com Veto}

O método aditivo com veto que compõe a família dos métodos de critério único de síntese foi proposto por De ALMEIDA (2013) com o objetivo de esclarecer uma situação em que um determinado decisor envolvido no processo decisório não aceita uma dada alternativa, pois em um determinada situação uma alternativa pode ser que acabe ficando muito abaixo mediante um dado critério considerado importante e ainda assim terminar ficando bem posicionada no ordenamento final das alternativas, Por isso, tem-se em vista que um resultado negativo pode ser compensado por um resultado positivo em determinado critério que causaria uma compensação do mal resultado desta alternativa em outro critério (TURET, 2015).

Deste modo, (DE ALMEIDA, 2013) propõe a realização de uma penalização intracritério para que desta forma, seja possível diminuir os problemas causados por consequência do efeito compensatório intercritério onde para identificação do veto são utilizados dois limiares para um dado critério: 
- $\mathrm{u}_{\mathbf{i}}=$ Limite superior, é determinado o valor mínimo aceitável de desempenho esperado $\mathrm{V}_{\mathrm{i}}$ (a) (sendo a uma alternativa qualquer) para dado critério em qualquer alternativa. Desta forma, o critério com este valor ou acima dele não irá penalizar a alternativa.

- $l_{\mathbf{i}}=$ Limite inferior, é determinado um valor máximo de desempenho vi(a). Logo, qualquer valor igual a este será penalizado, independente do desempenho de outros critérios analisados.

A função $Z_{i}(a)$ descreve o veto para a alternativa "a"

$$
z i(a)=\begin{gathered}
0, \\
1, \\
\frac{v i(a)-l i}{u i-l i}
\end{gathered} \quad\left\{\begin{array}{c}
\text { if } v i(a) \leq l i \\
\text { if } v i(a) \geq u i \\
\text { if } l i \leq v i(a) \leq u i
\end{array}\right.
$$

Existem dois métodos aditivos com veto: o primeiro para o problema de escolha e o segundo para ordenação. Na problemática de ordenação o decisor está com interesse em elencar uma posição relativa de cada alternativa a um dado conjunto, a fim de não escolher apenas uma alternativa (TURET, 2015).

Logo, uma função ponderada é aplicada para cada critério i:

$$
r_{i}(a)=z_{i}(a) k_{i}
$$

Onde, $z_{i}(a)$ é obtido a partir de (1) e $k_{i}$ é a constante da escala " $i$ " para a qual a alternativa está limitada. $\mathrm{O}$ índice ponderado de veto é formado pelo somatório de todas as funções de veto obtidas em (2), logo temos que:

$$
r(a)=\sum_{i=1}^{n} r_{i}(a)
$$

Sendo assim, a função ponderada de veto, pode ser integrada à fórmula do método aditivo determinístico convencional, para obter a ordenação das alternativas:

$$
v(a)=r(a) \sum_{i=1}^{n} k_{j} v_{j}(a)(4)
$$




\section{Modelo Proposto}

O modelo de multicritério deste trabalho, foi desenvolvido acerca da complexidade de melhorar os resultados do setor de manutenção de uma empresa do setor têxtil do agreste de Pernambuco. Com o intuito de auxiliar o gestor no processo de tomada de decisão diante desta problemática. O modelo é voltado para um problema que envolva apenas um único decisor no processo.

Figura 1 - Modelo multicritério de apoio a decisão

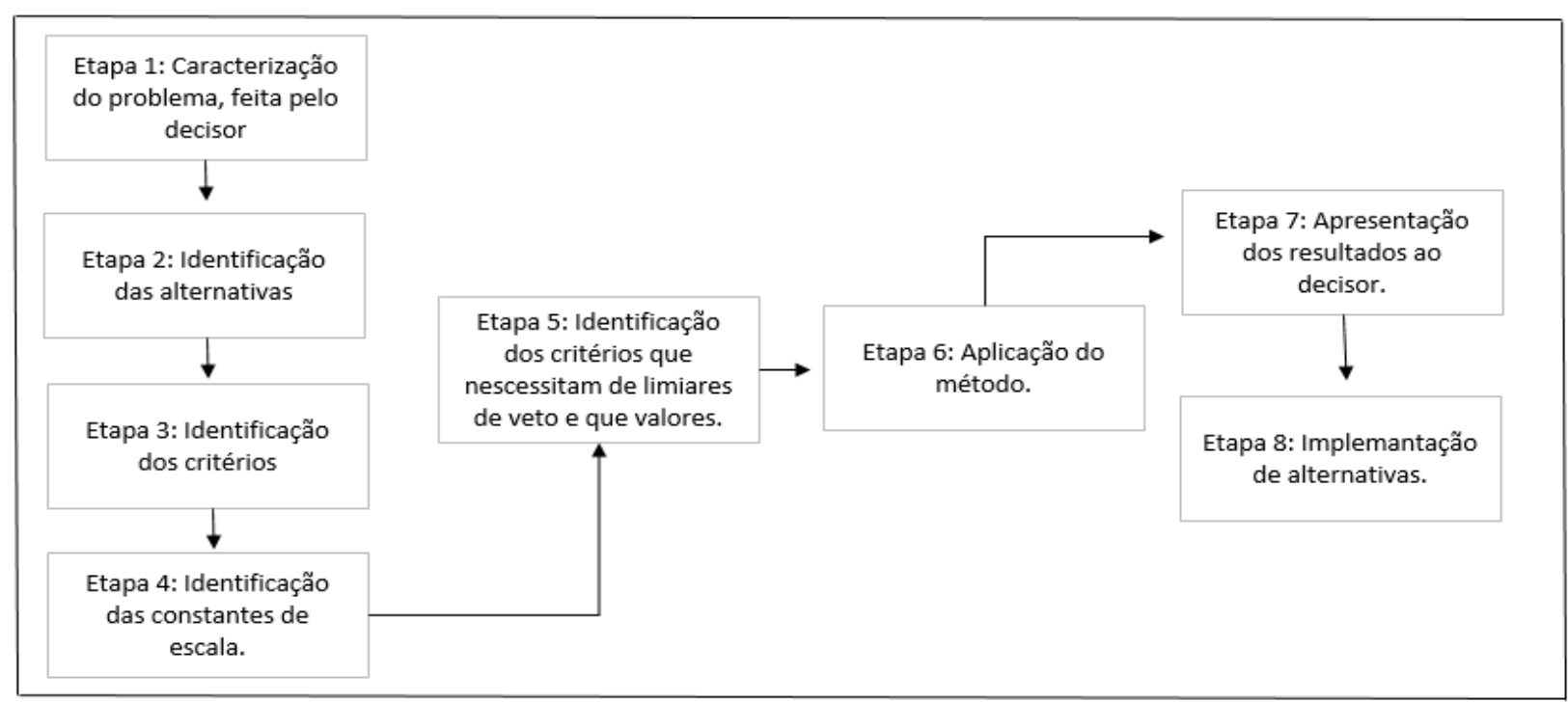

Fonte: Os Autores (2019)

Tem-se:

- Etapa 1: nesta etapa há a identificação dos principais desafios que o setor de manutenção da organização estudada apresenta. Esta fase é importante, pois a compreensão correta de tais desafios será um fator decisivo para o sucesso do modelo;

- Etapa 2: esta etapa envolve a identificação das alternativas que serão analisadas. Estas alternativas visam a melhoria dos resultados do setor de manutenção da empresa observada;

- Etapa 3: já neste momento ocorre a identificação dos critérios que devem ser utilizados na avaliação das alternativas. Os critérios devem ser o mais objetivos possível para que de tal forma que seja possível a avaliação do desempenho das alternativas; 
- Etapa 4: Com isso, assumindo o pressuposto de que a estrutura de preferência propicie ao decisor o uso do modelo aditivo com veto e que os critérios sejam mutualmente independentes em preferência, se faz necessário identificar as constantes de escala para cada critério, pois as constantes de escalas revelam o quanto o decisor estará disposto a perder em um critério em detrimento de outro;

- Etapa 5: nessa etapa identifica-se a necessidade de inclusão de veto ou não em algum critério ou alguns critérios, por isso caso seja necessário, será preciso identificar os limiares. Tais limiares serão usados como valores máximos e mínimos de uma determinada alternativa sobre um dado critério;

- Etapa 6: etapa em que ocorre a aplicação do modelo aditivo com veto para a problemática da escolha.

- Etapa 7: etapa onde os resultados são apresentados ao decisor;

- Etapa 8: etapa após a apresentação dos resultados ao decisor, fazendo com que, este possa implementar alternativas de ações.

A partir dessa escolha, o gestor analisa, por recomendação do decisor ou por constatar incoerências, que pode ser necessário tornar a etapas anteriores. Para isso, é imprescindível o entendimento das etapas do modelo por parte do decisor e a determinação na reavaliação de cada etapa seja qual for o período, de modo que toda a elaboração proposta esteja sob sua confiança.

\section{Aplicação Numérica}

O cenário exposto refere-se à uma empresa de confecção de roupas, situada no APL Têxtil do Agreste Pernambucano. O dono da empresa (decisor) enxergou fragilidades nas manutenções de suas máquinas, decorrentes da baixa produtividade e atraso nas entregas. Desse modo, a necessidade de tomar decisões para solucionar os problemas é imprescindível. Para isso, o analista surge como fonte de auxílio para o decisor encontrar o melhor caminho em busca de atingir suas necessidades.

Tendo como fundamento o modelo proposto na Figura 1, os pontos críticos foram observados e apontados como o foco do trabalho os seguintes desafios: manutenção preventiva e comunicação interna.

Partindo da descrição apresentada, alternativas de ação foram sugeridas para os desafios focais. Servindo como base para mudanças positivas nos resultados da empresa e melhoria no 
setor que apresenta dificuldades. Tais alternativas, expostas na Tabela 3, surgiram de pesquisas realizadas na literatura e em sites especializados em manutenção.

Tabela 3 - Alternativas de ação para melhoria dos resultados

\begin{tabular}{c|c|c}
\hline ID & Alternativas & Descrição \\
\hline \hline A1 & $\begin{array}{c}\text { Implementação das } \\
\text { reuniões de bom dia }\end{array}$ & $\begin{array}{c}\text { Reunião diária para informar as ações tomadas e } \\
\text { informar o andamento de outras }\end{array}$ \\
\hline A2 & Catalogar as máquinas & Identificando todas as peças e suas quantidades \\
\hline A3 & Identificar peças críticas & $\begin{array}{c}\text { Listagem das peças cruciais para o funcionamento do } \\
\text { equipamento }\end{array}$ \\
\hline A4 & $\begin{array}{c}\text { Gerir o estoque mínimo } \\
\text { das peças mais } \\
\text { importantes }\end{array}$ & $\begin{array}{c}\text { Identificando as peças e listando suas quantidades } \\
\text { mínimas para comprar os itens quando necessário e } \\
\text { manter o estoque }\end{array}$ \\
\hline A5 & $\begin{array}{c}\text { Instalar um quadro de } \\
\text { Gestão a Vista }\end{array}$ & $\begin{array}{c}\text { Realizando cotações para comprar com o melhor custo } \\
\text { benefício e então colocá-lo num local visível para todos }\end{array}$ \\
\hline A6 & $\begin{array}{c}\text { Treinar operadores e } \\
\text { líderes de produção }\end{array}$ & $\begin{array}{c}\text { Passando instruções básicas de bom uso das máquinas e } \\
\text { equipamentos, além de instruções de como agir diante } \\
\text { eventuais paradas }\end{array}$ \\
\hline
\end{tabular}

Fonte - Os Autores (2020)

Permitindo a realização do processo decisório, os critérios listados na Tabela 4 serviram de base para o decisor elicitar os referentes pesos para o modelo ser aplicado. Os valores para cada alternativa e as constantes de escala utilizada estão descritas na matriz de avalição na Tabela 5.

Tabela 4 - Critérios analisados no estudo

\begin{tabular}{|c|c|c|c|}
\hline ID & Critérios & Escala & Descrição \\
\hline $\mathrm{C} 1$ & $\begin{array}{c}\text { Custo de } \\
\text { Implementação }\end{array}$ & Monetária & $\begin{array}{c}\text { Preço para implementar a } \\
\text { alternativa }\end{array}$ \\
\hline $\mathrm{C} 2$ & $\begin{array}{c}\text { Tempo de } \\
\text { Implementação }\end{array}$ & Dias & $\begin{array}{c}\text { Tempo para implementar a } \\
\text { alternativa }\end{array}$ \\
\hline $\mathrm{C} 3$ & Custo de Manutenção & Monetária & $\begin{array}{c}\text { Preço para implementar a } \\
\text { alternativa }\end{array}$ \\
\hline $\mathrm{C} 4$ & $\begin{array}{c}\text { Impacto no Tempo de } \\
\text { Parada }\end{array}$ & $\begin{array}{c}\text { Escala ordinal de três } \\
\text { pontos: } \\
\text { 1- Baixo; } \\
\text { 2- Médio; } \\
\text { 3- Alto. }\end{array}$ & $\begin{array}{c}\text { Resultado direto no tempo } \\
\text { entre paradas }\end{array}$ \\
\hline
\end{tabular}


Tabela 5 - Matriz de avalição

\begin{tabular}{c|c|c|c|c}
\hline & $\mathrm{C} 1$ & $\mathrm{C} 2$ & $\mathrm{C} 3$ & $\mathrm{C} 4$ \\
\hline \hline $\mathrm{ki}$ & 0,15 & 0,30 & 0,10 & 0,45 \\
\hline $\mathrm{A} 1$ & 300 & 30 & 50 & 3 \\
\hline $\mathrm{A} 2$ & 3500 & 90 & 500 & 2 \\
\hline $\mathrm{A} 3$ & 600 & 15 & 50 & 2 \\
\hline $\mathrm{A} 4$ & 2100 & 25 & 300 & 2 \\
\hline $\mathrm{A} 5$ & 450 & 30 & 50 & 1 \\
\hline A6 & 2500 & 21 & 200 & 3 \\
\hline
\end{tabular}

Fonte - Os Autores (2020)

Dando continuidade no processo, o decisor apontou dois critérios para serem vetados: C2 (Tempo de Implementação) e C4 (Impacto no Tempo de Parada). Para o C2, estabeleceu um limiar superior de 30 dias e para $\mathrm{C} 4$, um limiar inferior de escala 3. Utilizando-se dos dados apresentados, o modelo proposto torna-se apto para ser executado. Para tal, uma planilha eletrônica foi desenvolvida para auxiliar nos cálculos por meio dos critérios escolhidos pelo decisor, cujo resultado está contido na Tabela 6.

Tabela 6 - Ordenação das alternativas com e sem veto

\begin{tabular}{c|c|c|c}
\hline $\begin{array}{c}\text { Ranking } \\
\text { Sem Veto }\end{array}$ & $\begin{array}{c}\text { Alternativa } \\
\text { Sem Veto }\end{array}$ & $\begin{array}{c}\text { Ranking } \\
\text { Com Veto }\end{array}$ & $\begin{array}{c}\text { Alternativa } \\
\text { Com Veto }\end{array}$ \\
\hline \hline 0,261360544 & A1 & 0,153279269 & A6 \\
\hline 0,213686686 & A3 & 0,144944558 & A1 \\
\hline 0,174345914 & A5 & 0,144455916 & A3 \\
\hline 0,153279269 & A6 & 0,139730529 & A5 \\
\hline 0,109566044 & A4 & 0,109566044 & A4 \\
\hline 0,087761543 & A2 & 0,082873275 & A2 \\
\hline
\end{tabular}

Fonte - Os Autores (2020)

O comparativo entre os métodos é de fundamental importância para compreensão das vertentes que a empresa deve tomar. Conforme observado, houve uma troca entre quatro 
alternativas, e tal condição é justificada pelo papel tomado pelo decisor ao estipular seus critérios de veto, bem como suas condições para cada um deles.

Ao tomar de forma mais específica as necessidades requeridas pelo decisor, o método aditivo com veto apresenta a alternativa A6 (Treinar operadores e líderes de produção) como ação principal a ser iniciada para a obtenção de um resultado mais efetivo, ao passo que no método aditivo tradicional indicada a alternativa A1 (Implementação das reuniões de bom dia). Em seguida surgem as alternativas A1 (Implementação das reuniões de bom dia), A3 (Identificar peças críticas) e A5 (Instalar um quadro de Gestão a Vista) como sequência das ações, quando no método sem veto seriam as alternativas A3 (Identificar peças críticas), A5 (Instalar um quadro de Gestão a Vista) e A6. As duas últimas posições não sofreram modificações e ficaram com as alternativas A4 (Gerir o estoque mínimo das peças mais importantes) e A2 (Catalogar as máquinas).

Nesse sentido, pautados no modelo proposto e assegurados na eficiência do método, é recomendado que a empresa invista inicialmente no Treinamento de Líderes e Operadores, a fim de obter melhores resultados na operação diária, tendo em vista que lidam diretamente com as máquinas. Após, a Implementação das Reuniões de Bom Dia surgem como fator importante no alinhamento das atividades e acontecimentos entre os setores; que servirão de base para a próxima alternativa a ser tomada: Identificação das Peças Críticas. Neste ponto, o alinhamento possibilitará que todos tomem conhecimento do que é realmente imprescindível de estar presente no estoque da empresa para não ocorrer paradas de máquina por longas durações.

\section{Considerações Finais, Limitações e Trabalhos Futuros}

Por maiores ritmos de produção e sequência do trabalho realizados, as grandes indústrias tem dedicado maiores atenções às manutenções de suas máquinas. Nessa mesma linha de raciocínio, o setor têxtil também se volta para essa área em busca de alternativas satisfatórias para implementar, garantindo a qualidade de seus produtos e obtendo um melhor desempenho com a equipe de manutenção.

Tendo como referência os estudos presentes na literatura, pode-se utilizar do modelo aditivo com veto para problemática de ordenação definido por De Almeida (2013) para apontar com maior precisão os caminhos que o dono da empresa deve seguir para atingir as suas necessidades. Assim, a primeira atividade a ser executada é o Treinamento de Líderes e Operadores. 
No que diz respeito aos fatores limitantes, a análise não contemplou os critérios de custos e dessa forma, surgem como possibilidades para trabalhos futuros. Além do mais, metodologias diferentes por ser aplicadas para fins comparativos entre métodos e modelos, sempre pensando em cruzar dados para transformar em informação e assim, produzir conclusões mais detalhadas em prol da satisfação do decisor.

\section{REFERÊNCIAS}

ALMEIDA, A. T. (2013). Processo de decisão nas organizações: construindo modelos de decisão multicritério. São Paulo: Atlas.

Almeida, A.T. et al. (2015). Multicriteria and Multiobjective Models for Risk, Reliability and Maintenance Decision Analysis, International Series in Operations Research and Management Science. Springer, New York, NY.

DE ALMEIDA, Adiel Teixeira; COSTA, Ana Paula Cabral Seixas. Aplicações com Métodos Multicritério de Apoioa Decisão. Universitária UFPE, Recife, 2003.

De Almeida, A. T. (2013). Additive-veto models for choice and ranking multicriteria decision problems. Journal of Operational Research. v.30, n.6, p.1-20.

GIL, Antônio Carlos. Como elaborar projetos de pesquisa. 4. ed. São Paulo: Atlas, 2008.

GOMES, Luiz Flavio Autran Monteiro; MORENO JR., Valter de Assis; WOITOWICZ, Bernardo Barbosa Chaves; LUCAS, Solange Maria Fortuna. Uma abordagem multicritério para a seleção de ferramentas de Business Intelligence. Revista Eletrônica de Sistemas de Informação, v. 10, n. 2, p. 1-28, 2011.

KARDEC, Alan; NASCIF, Júlio. Manutenção: função estratégica, Rio de Janeiro, ed. Qualitymark, 1998.

KENNEY, R. L. e RAIFFA, H. (1976). Decision with multiple objectives: preferences and value trade-offs. New York: John Wiley.

MOREIRA, Miguel Ângelo Lellis; SANTOS, Marcos dos; GOMES, Carlos Francisco Simões. Desenvolvimento de Ferramenta Computacional em Python: implementação e estudo de caso dos métodos PROMETHEE I, II e III. In: SIMPÓSIO DE PESQUISA OPERACIONAL

E LOGÍSTICA DA MARINHA, 19., 2019, Rio de Janeiro, RJ. Anais [...]. Rio de Janeiro: Centro de Análises de Sistemas Navais, 2019.

NEVES, Roberta Braga; PEREIRA, Valdecy and COSTA, Helder Gomes. Auxílio multicritério à decisão aplicado ao planejamento e gestão na indústria de petróleo e gás. Produção. 2015, vol.25, n.1, pp.43-53. Epub Sep 03, 2013.

SANTOS, Antonio Raimundo dos. Metodologia científica: a construção do conhecimento. 3. ed. Rio de Janeiro: DP\&A editora, 2000.

SHAMBLIN, James E. \& STEVENS JR, G.T. . Pesquisa Operacional - Uma Abordagem Básica; editora Atlas, São Paulo/SP; p. 13; 1979

SLACK, Nigel; CHAMBERS, Stuart; JOHNSTON, Robert. Administração da Produção. 2. ed. São Paulo: Atlas, 2000. 747 p.

Thiollent, M. (2009). Metodologia de Pesquisa-ação. São Paulo: Saraiva

Turet, J.G. (2015). Modelo de apoio a decisão para plano de ação em empresas m-commerce. Dissertação UFPE.. 